\title{
Domain decomposition for 3D electromagnetic modeling
}

\author{
Zonghou Xiong \\ CRC AMET, Earth Science, Macquarie University, Sydney, NSW 2109, Australia
}

(Received November 2, 1998; Revised August 16, 1999; Accepted August 23, 1999)

\begin{abstract}
Using the staggered grid full domain 3D modeling schemes of various accuracies have been developed. This study focuses on the second order finite difference method with the 13-point rule for meshes extending into the air. Tests with Krylov space iterative solvers indicate that the restarted Bi-CG Stablised method offers the best convergence for our problems. Because the air and the conductive earth have distinctive physical properties which greatly broaden the spectra of the whole matrix system, the whole mesh with both domains in one system either converges very slowly or fails to converge completely. However, the matrix systems for each domain have much smaller condition numbers. To overcome instability caused by the inclusion of the air in the mesh a domain decomposition method are experimented. Tests show that the adaptive iteration amongst the subdomains converges exponentially, which implies that large models can be solved by using the domain decomposition method.
\end{abstract}

\section{Introduction}

Electromagnetic modeling based on staggered grids, or Yee-grids (Yee, 1966), has proven to be an effective way of solving the scattering problems in geophysical problems both in the time and in the frequency domain. Evaluating either the electric or the magnetic field on the edge of the cells rather than on corner nodes, staggered grid methods are also special forms of more general methods, the edge element methods, which are capable of handling high (conductivity or permeability) contrasts models (Bossavit, 1988a,b; Mayergoyz and D’Angelo, 1993; Mur, 1994; Sugeng, 1998).

While time domain finite difference (FDTD) methods (Wang and Hohmann, 1993) avoids solving a matrix equation at the expense of lengthy time-stepping procedures, frequency domain methods involve solutions of large sparse matrix systems. The spectral decomposition method (Druskin and Knizhnerman, 1988, 1994; Árnason, 1998) utilizes the special structure of the discrete forms of Maxwell's equations in conductive regions to obtain efficient solutions both in the time and in the frequency domain. For a solution mesh including the air region, the resulting matrix can be very ill-conditioned. Iterative solutions of such a matrix system are indeed very slow and correction schemes such as divergence corrections (Smith, 1996a; Mackie et al., 1994; Weidelt, 1998) are necessary to speed up convergence.

Contrasts in physical properties, such as the conductivities of the earths, increase the condition number of the matrix systems and slow down the iterative solvers. With zero conductivity the inclusion of the air in the numerical grids can pose substantial difficulties in the solution of the matrix system. At low frequencies when displacement currents are neglected, the air can be excluded in the computation using the field values at the air-earth interface, either by integral

Copy right (C) The Society of Geomagnetism and Earth, Planetary and Space Sciences (SGEPSS); The Seismological Society of Japan; The Volcanological Society of Japan; The Geodetic Society of Japan; The Japanese Society for Planetary Sciences. boundary conditions (Wang and Hohmann, 1993; Druskin and Knizhnerman, 1994; Weaver et al., 1995; Agarwal and Weaver, 1998), or by matrix-valued exponential functions (Árnason, 1998). However, in airborne applications, grids extending to the air region have to be considered.

To find optimized schemes using the staggered grid, various methods of $2 n d$ and $4 t h$ order accuracies (Xiong et al., 1996; Xiong 1998) have been devised. Higher order methods do yield higher accuracy for whole space models without conductivity heterogeneities, although the resulting matrices have wider bandwidths and higher condition numbers. This agrees with the results of Shlager et al. (1993). However, the advantage of higher accuracy with higer order schemes appears to be less obvious if the medium becomes inhomogeneous. This is due to the fact that the discrete forms of Maxwell equations require averaging conductivities around grid cells. Thus the accuracy of a scheme involving homogeneous conductivities depends mostly on the sizes of the cells, instead of the order of accuracy of the approximation of the Maxwell equations. Nevertheless, mixed use of $4 t h$ order rules for homogeneous regions and $2 n d$ order rules for inhomogeneous regions may become attractive for large models.

Since the inclusion of regions of different physical properties into one domain generally broadens the spectra of the matrix system and slows down convergence of iterative solvers, it is natural to decompose these regions into different subdomains and to find a solution to the whole system by taking into account their mutual interactions. Domain decomposition can also be used to form effective preconditioners. In geo-electromagnetic applications, grids extending into the air cause difficulties for iterative solvers. I tried to separate the air and the earth by two domains. Solutions of the matrix systems within individual domains converges drastically faster, by as much as a factor of 10 , than the whole system containing them both. In this paper I will show that 
by dividing the whole mesh into two to three subdomains the adaptive iteration amongst the domains converge exponentially, indicating great potential for modeling very large models.

\section{Basic Equations}

Maxwell equations for electromagnetics read:

$$
\begin{aligned}
\nabla \times \mathbf{E} & =-i \omega \mu \mathbf{H}, \\
\nabla \times \mathbf{H} & =\underline{\sigma} \cdot \mathbf{E}+\mathbf{J}^{i m p},
\end{aligned}
$$

where $\mathbf{J}^{i m p}$ is the impressed excitation current, and $\underline{\sigma}$ is the conductivity of the medium which can be anisotropic.

On staggered grids either the electric components are put on the cell edges with the magnetic components on the cell facet, or vice versa. This decouples the components of the electromagnetic field into triplets on Cartesian coordinate systems. Figure 1 shows the relation of the components in the second order finite difference equation for the component $E_{z}$. The facet magnetic components are removed by their relations with the electric components on the edges, or Eq. (1), which lead to a 13-point $O^{2}$ rule. Conductivities required by Eq. (2) are the averaged conductivities around the nodes of the eletric components. The matrix system is in general non-symmetric with complex diagonal entries containing the averaged conductivities, although the matrix due to $O^{2}$ approximation can be scaled to a symmetric form (Smith, 1996a). Higher order approaches can be obtained by applying higher order interpolation rules. In the following discussion I shall focus on the matrix system obtained by the 13-point $O^{2}$ rule.

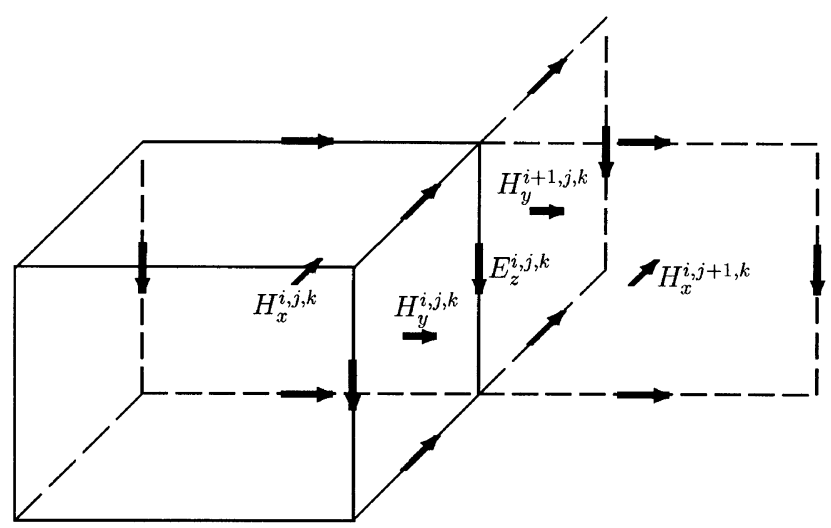

Fig. 1. Relations for the vertical electric components of staggered grid cells. The complete cell in solid lines is indexed as $(i, j, k)$. Only components related to the equation for $E_{z}^{i, j, k}$ are shown. The facet magnetic components are removed by their relations with the electric components on the edges. This lead to the 13-point $O^{2}$ rule.

\section{A Robust Iterative Solver}

The matrix equation obtained by the 13-point rule is highly ill-conditioned. Most Krylov-subspace methods are slow to converge with this type of matrices. I have tried a few solvers for non-symmetric matrices which do not require transposes, in particular, CGS, Bi-CGSTAB, and GMRES.

Because of the excessive storage requirement, restarted version of GMRES is necessary. But the restarted GMRES did not converge well and thus it was not pursued any further CGS method is a variant of BiCG that applies the contraction operator twice. This increases the convergence rate but causes high irregularities which in the end may fail to converge at all. Bi-CGSTAB is designed to overcome these irregularities. However, this smooth convergence often stagnates after certain iterations, unable to reduce the residual any further and fail to converge. To overcome this stagnation, Sleijpen and Fokkema (1993) introduced a restarted BiCGSTAB method, Bi-CGSTAB $(l)$. This method combines a $l$-step restarted GMRES method and Bi-CG. Bi-CGSTAB $(l)$ does not require the transpose matrix and stores a smaller number of vectors than the restarted GMRES method. Tests show that Bi-CGSTAB $(l)$ is very efficient in our applications. CGS and Bi-CGSTAB often fail to converge at low frequencies, but Bi-CGSTAB $(l)$ does not exhibit this problem and always converges. Figure 2 shows the convergence patterns of CGS, Bi-CGSTAB and Bi-CGSTAB $(l)$ with $l=2$ and $l=4$ for a $20 \times 20 \times 20$ non-uniform mesh with a $3 \mathrm{D}$ conductor in a half-space.

It is anticipated that preconditioners improve the convergence of our iterative solvers. It is found that the effects of preconditioning varies greatly from solver to solver. Incomplete LU decomposition (ILU) and block diagonal decomposition of various block sizes have been tested. The most straightforward test on ILU is to store the banded sparse matrix as a full matrix, do full LU decompositions, and discard those elements in the factorized matrix which correspond to the zero elements of the original matrix. This can be done for a mesh of say, $10 \times 10 \times 10$ cells. Our tests with such preconditioning ILU matrices did not seem to show expected improvements in convergence, although imcomplete Cholesky decompositions were reported to be successful in 3D MT modeling (Mackie et al., 1994; Smith, 1996b).

Unknowns in the mesh can be grouped so that elements of the system matrix form clusters of blocks near the diagonal. This is done by grouping cells in the indexing sequence. This allows the use of block diagonal matrices as preconditioners. The most common diagonal blocks are the three-diagonal blocks which involve three components of each single cell. This $3 \times 3$ block-diagonal preconditioner seems to be very effective for the CGS and Bi-CGSTAB method, better than Jacobian preconditioner that uses the diagonal elements only. However, in many cases it still could not help bring the solution to convergence.

Block diagonal submatrices bigger than 3 by 3 contain non-zero elements. To study the effect of preconditioning, I tried block diagonal matrices of various sizes, by grouping a number of cells together to maximize the number of non-zero elements in the block diagonal matrices. Groups of about $4 \times 4 \times 4$ cells, which yield block diagonal matrices of dimension of $1728 \times 1728$ (involving $12 \times 12 \times 12$ unknowns) improves the convergence at the cost of factorizing a submatrices with many non-zero elements. More brutal tests are done by truncating the original whole sparse matrix for elements at certain distance away from the diagonal. This is feasible for a mesh of, say $10 \times 10 \times 10$. The truncated matrix is then LU decomposed to be used as the preconditioner. Our tests show that significant increase in convergence rate can be obtained only if the preconditioner is about 80 percent 


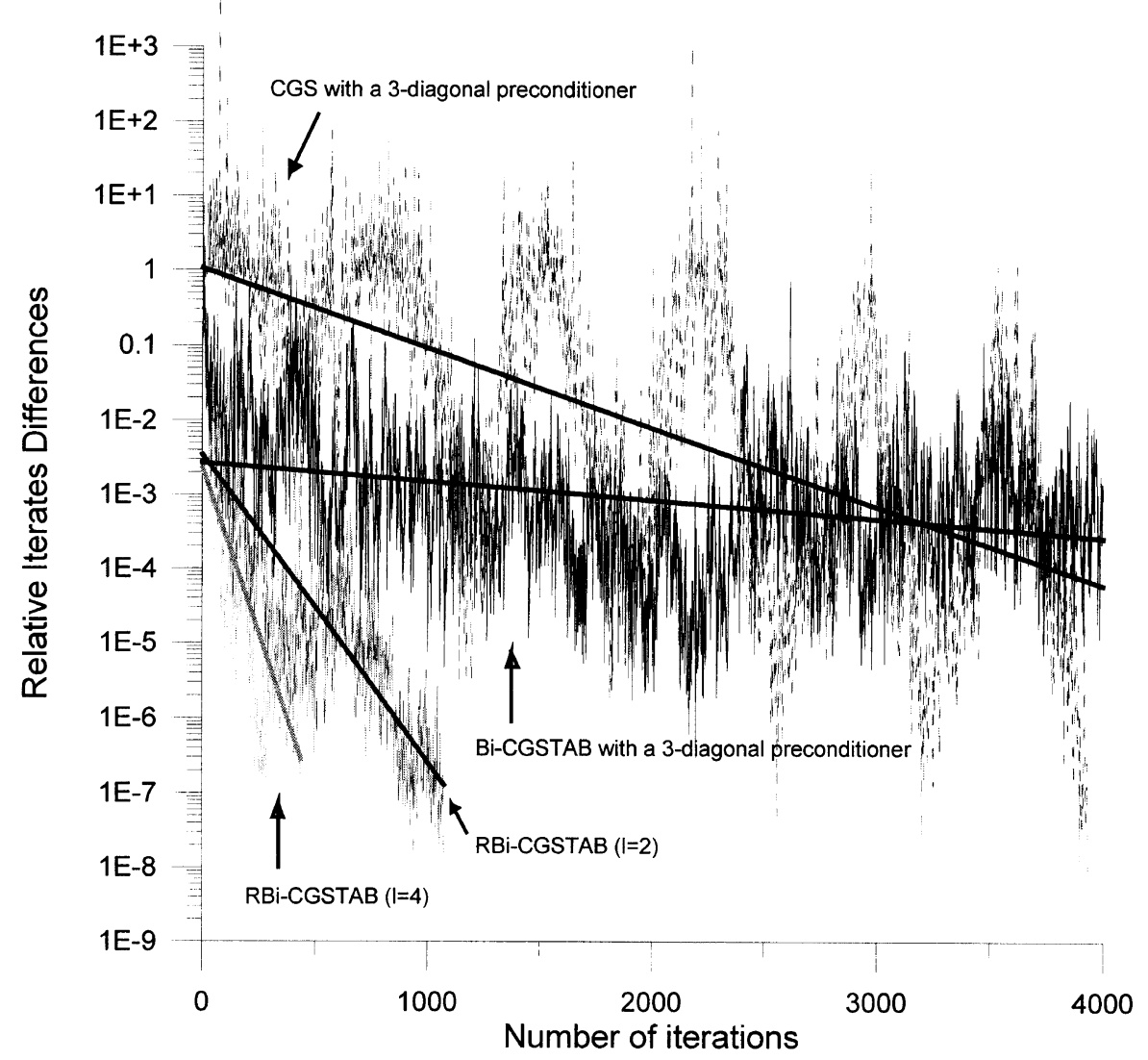

Fig. 2. Convergence patterns of CGS, Bi-CGSTAB and Bi-CGSTAB $(l)$ for a $20 \times 20 \times 20$ non-uniform mesh with a $3 \mathrm{D}$ conductor in a half-space. $\operatorname{Bi}-\operatorname{CGSTAB}(l)$ shows superior performances for all models we tested.

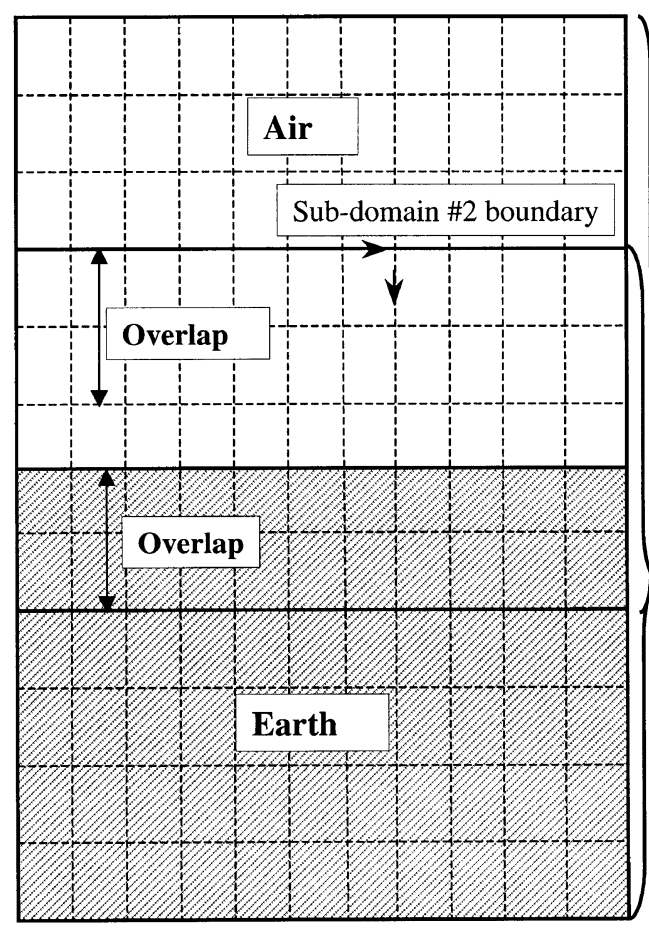

Fig. 3. Two overlapping subdomains.
Subdomain \#1

Subdomain \#2

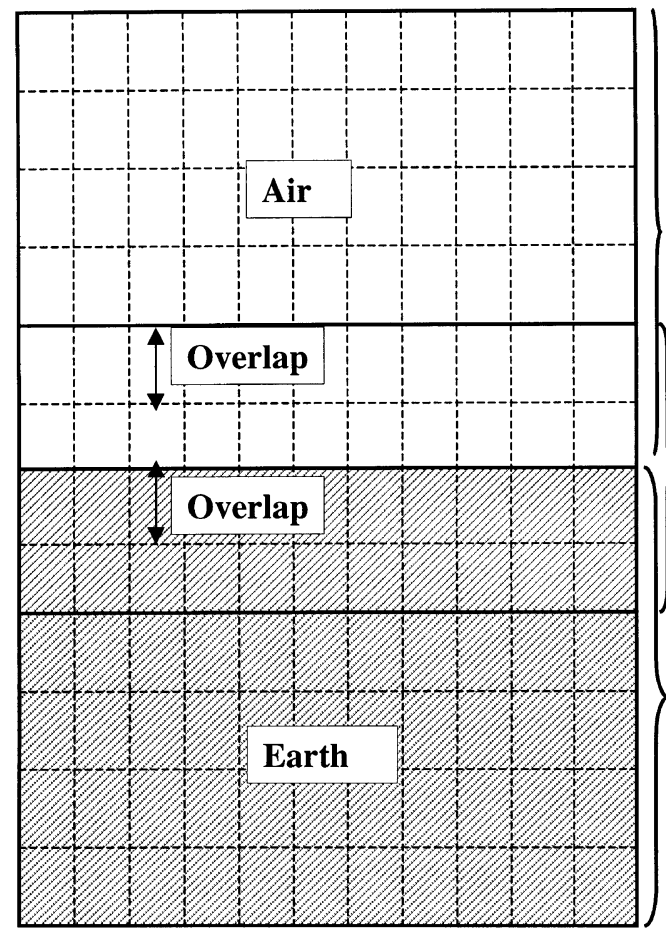

Subdomain \#1

Subdomain \#2

Subdomain \#3

Fig. 4. Three overlapping subdomains. 


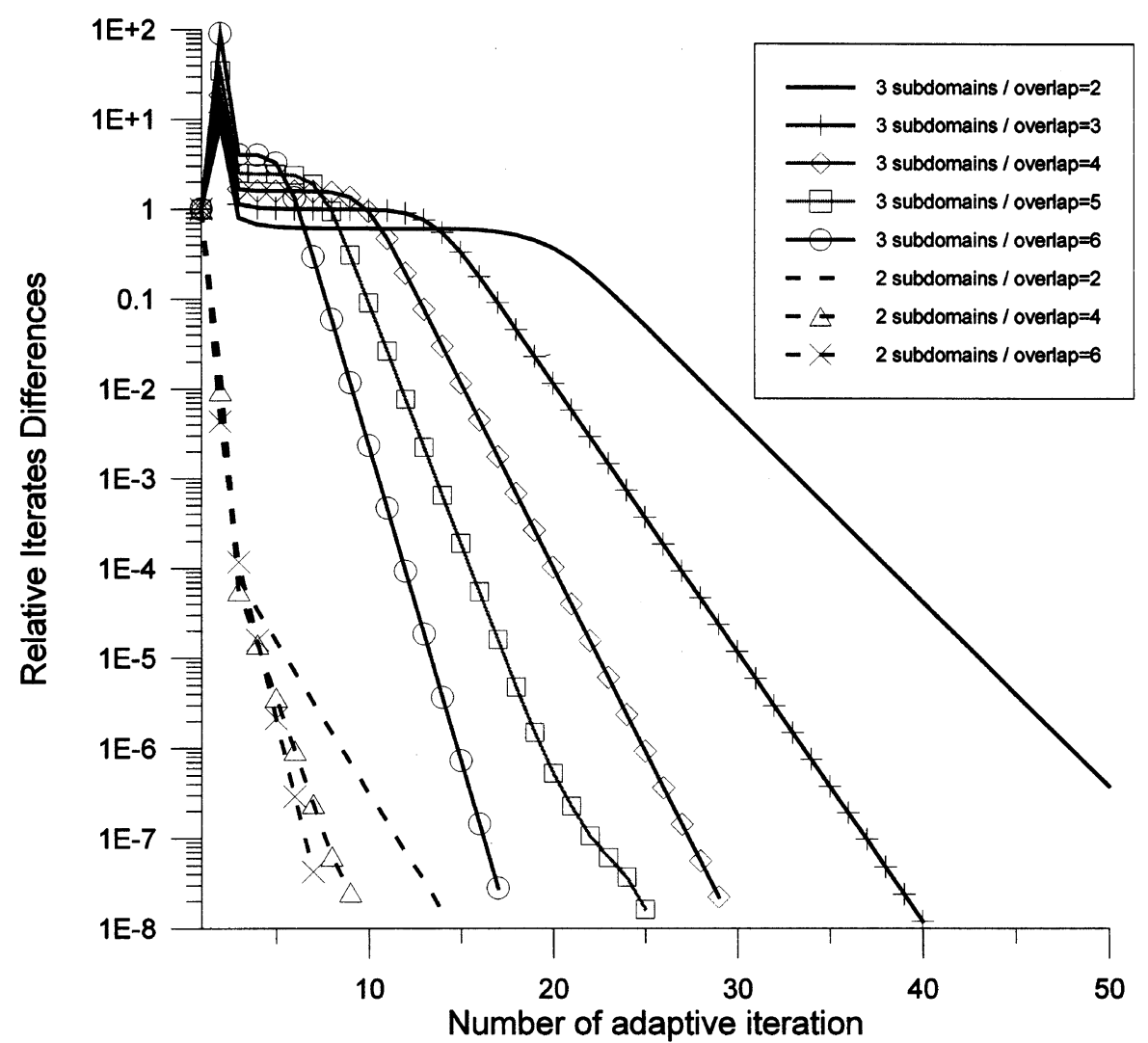

Fig. 5. Convergence of adaptive iteration for a $20 \times 20 \times 20$ mesh with a $3 \mathrm{D}$ conductor in a half space. After certain iterations solutions converge at exponential rates.

of the original matrix, or $a_{i j}=0,|i-j|>0.8 n, n$ being the dimension of the whole matrix. These tests indicate that block diagonal preconditioners with more than 3-diagonals are practically useless. The tests with the truncated matrices also show that for a preconditioner to be effective, it must be very close to the original matrix.

\section{Domain Decomposition with Adaptive Iteration}

Domain decomposition methods in general fall into two categories, namely non-overlapping methods or Schur complement methods and overlapping methods or Schwarz methods. Schur complement methods are mostly used as preconditioners (Larsson, 1999). Schwarz methods for overlapping subdomains can be used as preconditioners as well as solvers to the matrix equations. I will apply a Schwarz method with adaptive iterations to solve the matrix system.

Assume that the mesh is decomposed into two subdomains, as shown in Fig. 3. Here overlap is introduced in the domains. I shall define the parameter overlap used in this study in a precise way. Suppose each rectangular grid block in Figs. 3 and 4 represent a cell. Figure 3 shows the case of overlap $=2$ and Fig. 4 shows the case of overlap $=1$. There are in fact $2 \times$ overlap +1 common cells between two subdomains. Of them $2 \times$ overlap are considered overlapping. There is always a cell that couples the two adjacent subdomains naturally without overlapping.

For subdomain \#1 the tangential components on the subdomain boundaries are used as given boundary values. For boundary components of subdomain \#2 I use all tangential components on the subdomain boundary plus the vertical components of the first layer of cells from the top boundary. This becomes the natural partition of the domains if there is no overlapping. Decompose the mesh into more domains can be done similarly, as is the three subdomains of Fig. 4.

To find the solution to the whole system in an adaptive fashion the initial values to the subdomain boundaries, say for subdomain \#1 are assigned. Then the electric field on the interior nodes is solved with the Bi-CGSTAB $(l)$ method. Since the top boundary of subdomain \#2 resides in the interior part of subdomain \#1, the field components get updated from the solution of the subdomain \#1. With these new boundary values the interior unknowns of subdomain \#2 are solve. This also updates the boundary values of subdomain \#1. This process is repeated until the iterates differences are smaller than a given level.

Figure 5 shows the adaptive iteration of the two and three subdomains. The model is a $300 \times 300 \times 200 \mathrm{~m}^{3}$ conductor of $1 \Omega \mathrm{m}$ buried $100 \mathrm{~m}$ below the surface of the ground. The mesh is $1.3 \times 1.3 \times 1.6 \mathrm{~km}^{3}$, discretized into $20 \times 20 \times 20$ cells, with the minimal cell dimension being $50 \times 50 \times 50$ $\mathrm{m}^{3}$. The mesh includes a $800 \mathrm{~m}$ thick air layer. The source is a current bipole of with a length of $58 \mathrm{~m}$ located at $(280,250$, $0)$. The coordinate center is on the ground over the center of the $3 \mathrm{D}$ conductor. Frequency is $300 \mathrm{~Hz}$. Halfspace primary field of the bipole is used at mesh boundaries. There is a common feature in the results shown in Fig. 5, namely the exponential convergences of the adaptive iterations both for the two subdomain case and for the three subdomain case. 


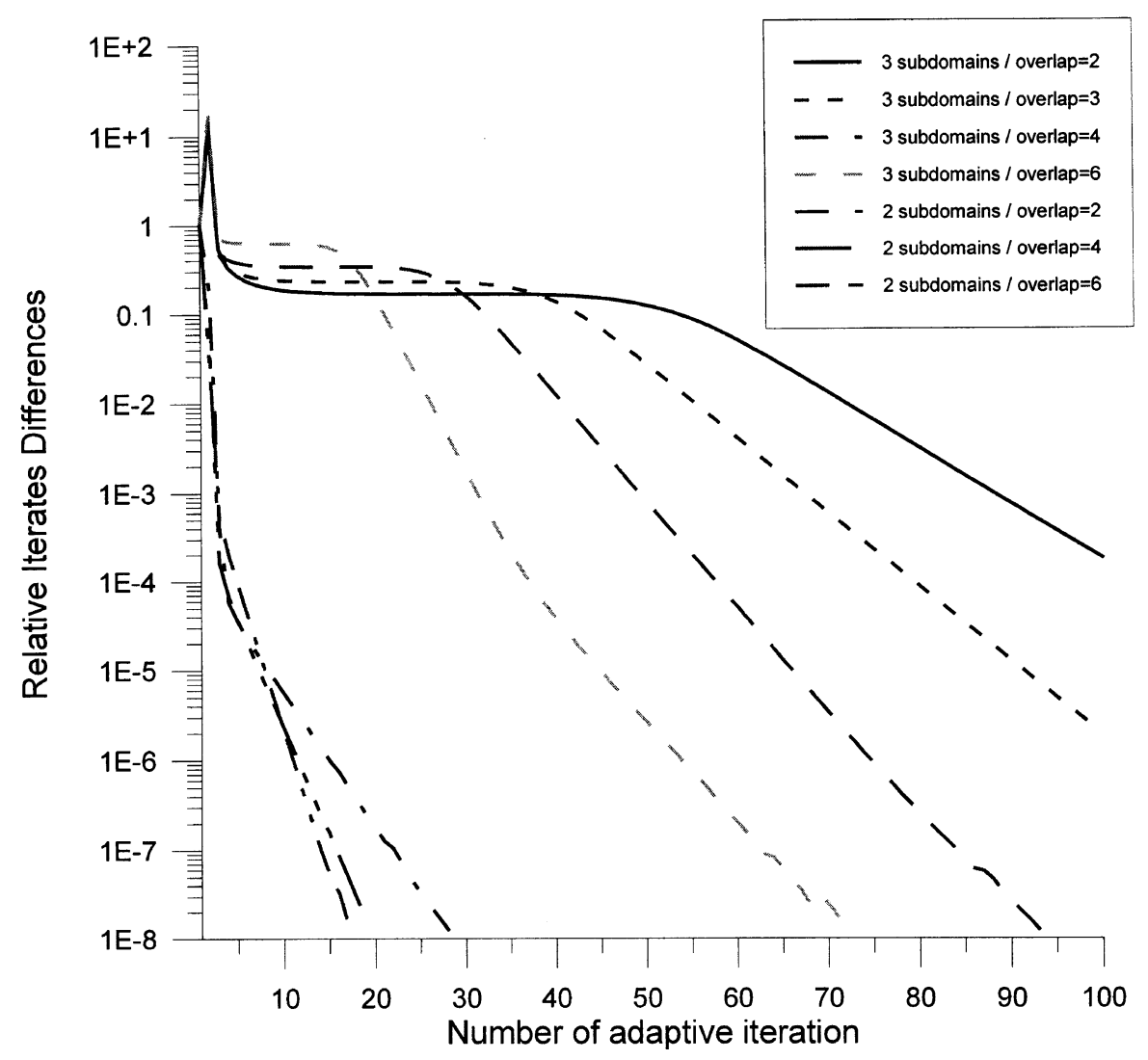

Fig. 6. Convergence of adaptive iteration for a $30 \times 30 \times 30$ mesh with a $3 \mathrm{D}$ conductor in a half space. The model is a $400 \times 600 \times 200 \mathrm{~m}{ }^{3}$ prism of $1 \Omega \mathrm{m}$ buried $50 \mathrm{~m}$ deep in the earth. The transmitter is an electric bipole at $300 \mathrm{~Hz}$. The mesh $2.5 \times 2.8 \times 2.5 \mathrm{~km}^{3}$ including $1.2 \mathrm{~km}$ air layer. Minimal cell size is $50 \times 50 \times 25 \mathrm{~m}^{3}$. Convergence is similar to Fig. 5 .

Overlapping is also very important to the adaptive iteration. Clearly more overlapping means faster convergence, albeit at the costs of increased subdomain sizes. Figure 6 with somewhat bigger mesh of $30 \times 30 \times 30$ also exhibits similar characteristics of convergence.

\section{Concluding Remarks}

The domain decomposition results presented above are successful tests showing the promising potential of the domain decomposition methods to our modeling problems. The exponential convergences of the adaptive iteration indicate that large systems can be broken into many subdomains and be solved in an adaptive fasion. However, the total run time to solve the system via the above procedure is higher than solving the whole system with an efficient solver such as Bi$\operatorname{CGSTAB}(l)$, because the subsystems for the subdomains are solved iteratively. Further work are needed to find effecitve preconditioners for the subdomains to minimize the solution time solving the subsystem.

Acknowledgments. The author wishes to thank Dr. Art Raiche for many valuable discussions. This work was supported by AMIRA project P223D. I wish to thank BHP Minerals, Billiton SA, Cominco Exploration, the Geological Survey of Finland, Geological Survey of Denmark \& Greenland, Geoterrex-Dighem, ISCOR, M.I.M. Exploration, North, Pasminco Exploration, Rio Tinto Exploration, Sumitomo Metal Mining Oceania, and Western Metals for their sponsorship of this project.

\section{References}

Agarwal, A. K. and J. T. Weaver, Comparison of electric fields obtained from 3D magnetic field calculations on fixed staggered grids, in The 14th Workshop on Electromagnetic Induction in the Earth, Sinaia, Romania, Aug. 16-22, 1998.

Árnason, K., A consistent discretization of the electromagnetic field in conducting media and application to the TEM problem, in $3 D$ Electromagnetics, edited by M. Oristaglio and B. Spies, Society of Exploration Geophysicists, 1998.

Bossavit, A., Whitney forms: a class of finite elements for three-dimensional computations in electromagnetism, IEE Proc., 135, 493-499, 1988a.

Bossavit, A., A rationale for "edge-elements", IEEE Trans. Magnetics, 24, 74-79, 1988b.

Druskin, V. and L. Knizhnerman, A spectral semi-discrete method for numerical solution of 3-D non-stationary problems in electrical prospecting, Phys. Sol. Earth, 24, 641-648, 1988.

Druskin, V. and L. Knizhnerman, Spectral approach to solving threedimensional Maxwell's diffusion equations in the time and frequency domain, Radio Sci., 29, 937-953, 1994.

Larsson, E., A domain decomposition method for the Helmholtz equation in a multilayer domain, SIAM J. on Scientific Computing, 20, 1713-1731, 1999.

Mackie, R. L., J. T. Smith, and T. R. Madden, Three-dimensional electromagnetic modeling using finite difference equations: The magnetotelluric example, Radio Sci., 29, 923-935, 1994.

Mayergoyz, I. D. and J. D'Angelo, A new point of view on the mathematical structure of Maxwell equations, IEEE Trans. Magnetics, 29, 1315-1320, 1993.

Mur, G., Edge elements, their advantages and their disadvantages, IEEE Trans. Magnetics, 30, 3552-3557, 1994.

Shlager, K. L., J. G. Maloney, S. L. Ray, and A. F. Peterson, Relative accuracy of several finite-difference time-domain methods in two and three dimensions, IEEE Trans. Antennas Propagat., 41, 1732-1737, 1993. 
Sleijpen, G. L. G. and D. R. Fokkema, BiCGSTAB(L) for linear matrices involving nonsymmetric matrices with complex spectrum, ETNA, 1, 1132, 1993.

Smith, J. T., Conservative modeling of 3-D electromagnetic fields; Part I: Properties and error analysis, Geophysics, 61, 1308-1318, 1996a.

Smith, J. T., Conservative modeling of 3-D electromagnetic fields; Part II: Biconjugate gradient solution and an accelerator, Geophysics, 61, 13191324, 1996b.

Sugeng, F., Modelling the transient responses of complex 3D geological structures using the 3D full-domain hexahedral edge-element finiteelement technique, in The 14th Workshop on Electromagnetic Induction in the Earth, Sinaia, Romania, Aug. 16-22, 1998.

Wang, T. and G. W. Hohmann, A finite-difference, time-domain solution for three-dimensional electromagnetic modeling, Geophysics, 58, 797-809, 1993.

Weaver, J. T., Agarwal, A. K., and X. H. Pu, Recent developments in three-dimensional finite difference modelling of the magnetic field in geo-electromagnetic induction, in International Symposium on Three- dimensional Electromagnetics, Schlumberger-Doll Research, Ridgefield, Connecticut, USA, Oct. 4-6, 1995.

Weidelt, P., Three-dimensional conductivity models: implications of electrical anisotropy, in 3D Electromagnetics, edited by M. Oristaglio and B Spies, Society of Exploration Geophysicists, 1998.

Xiong, Z., A study of high accuracy methods for full-domain 3D electromagnetic modelling, in The 14th Workshop on Electromagnetic Induction in the Earth, Sinaia, Romania, Aug. 16-22, 1998.

Xiong, Z., A. Raiche, and F. Sugeng, A high accuracy staggered grid Galerkin method for 3D electromagnetic modelling, in The 13th Workshop on Electromagnetic Induction in the Earth, Onuma, Japan, July-1218, 1996.

Yee, K. S., Numerical solution of initial boundary value problems involving Maxwell's equations in isotropic media, IEEE Trans. Antennas Propagat., AP-14, 302-307, 1966.

Z. Xiong (e-mail: zxiong@laurel.ocs.mq.edu.au) 